\title{
A CONCISE ROUTE FOR THE ONE-POT MULTI-COMPONENT SYNTHESIS OF 4,6-DISUBSTITUTED 2-AMINOPYRIDINE-3-CARBONITRILES AND PYRANOPYRAZOLES USING COBALT (II) NITRATE HEXAHYDRATE AS CATALYST
}

\author{
Hamed PEJMAN, Nourallah HAZERI, ${ }^{*}$ Maryam FATAHPOUR and Homayoun FAROUGHI NIYA \\ Department of Chemistry, Faculty of Science, University of Sistan and Baluchestan, P.O. Box 98135-674, Zahedan, Iran
}

\begin{abstract}
Three facile and efficient routes for one-pot, cobalt (II) nitrate hexahydrate catalyzed multi-component synthesis of 2-amino-3cyanopyridine and pyranopyrazole derivatives have been developed. The present protocols offer the products in good yields from the simple and readily available starting materials. The other salient features of these protocols are operational simplicity, easy isolation of product without the need of column chromatographic purification, and the use of cobalt (II) nitrate hexahydrate as an efficient catalyst.
\end{abstract}

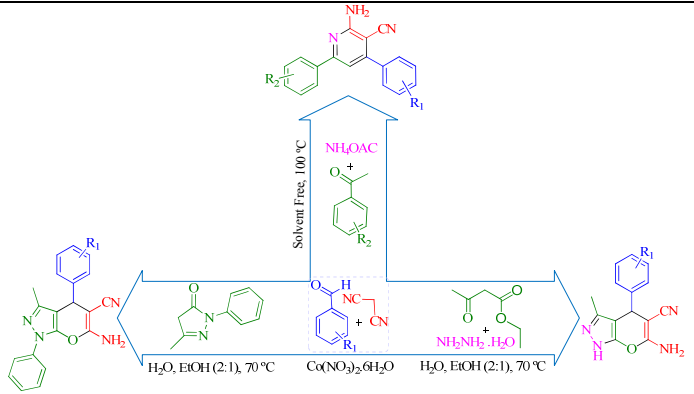

\section{INTRODUCTION}

Heterocyclic skeleton, specially functionalized heterocycles exist in many important compounds with unique biological activities. They occupy a vast scope in the drug discovery process. Hence, explore topics on the synthesis of heterocyclic motifs has gained special attention. ${ }^{1-3} \mathrm{~N}$ heterocycles are highly desirable compounds because of their differing chemical and pharmacological significance. Of these, pyridine and pyrazole derivatives are appeared as important building blocks due to a wide range of biological profiles. Cyanopyridine derivatives possess a wide variety of bioactivities (e.g. $\mathrm{A}_{2 \mathrm{~A}}$ adenosine receptor antagonists, ${ }^{4}$ IKK- $\beta$ inhibitors, ${ }^{5}$ and potent inhibitor of HIV-1 integrase ${ }^{6}$ ). They have several pharmaceutical activities (e.g. Antibactrial, ${ }^{7}$ anticancer, ${ }^{8}$ anticardiovascular ${ }^{9}$, antiinflammatory, analgesic, and antipyretic ${ }^{10}$ ). Pyrazole unit is widely prevalent in natural products and bioactive molecules. ${ }^{11,12}$ They act as essential structures in the skeletons of numerous commercial drugs (e.g. celecoxib, ENMD-2076, R1530, PNU-32945, rimonabant, metamizole, and sulfaphenazole). ${ }^{13-16}$ Cyanopyridine and pyrazoles are also valuable intermediates in organic synthesis. ${ }^{17,18}$ In recent years, many synthetic approaches for preparation of 2-aminopyridine-3-carbonitriles and pyranopyrazoles have been developed; each has its advantages and disadvantages, namely longer reaction time, toxic solvents, harsh reaction conditions and low yields.

In a continuation of our endeavors towards the development of synthetic methodology, ${ }^{19-23}$ here we reported a one-pot four-component route for synthesis of 2-aminopyridine-3-carbonitriles from reaction between arylaldehydes $\mathbf{1}$, malononitrile $\mathbf{2}$, ketones 3, and ammonium acetate $\mathbf{4}$ and also an efficient one-pot, three/four-component protocol

\footnotetext{
*Corresponding author: Tel/ fax:+985412446565; E-mail: nhazeri@chem.usb.ac.ir,n_hazeri@yahoo.com
} 
for synthesis of dihydropyrano[2,3-c]pyrazoles by condensation of arylaldehydes $\mathbf{1}$, malononitrile $\mathbf{2}$, hydrazine monohydrate $\mathbf{6}$ and ethyl acetoacetate $\mathbf{7}$ or 5-methyl-2-phenyl-2,4-dihydro-3H-pyrazol-3one 9 in the presence of cobalt (II) nitrate hexahydrate as an effective catalyst in a benign conditions (Scheme 1).

\section{EXPERIMENTAL}

Melting points and FT-IR spectra of compounds were measured with an Electrotherma 19100 apparatus. The ${ }^{1} \mathrm{H}$ and ${ }^{13} \mathrm{C}$ NMR spectra of the compounds were recorded on a Bruker DRX-300 Avance instrument in DMSO at $300 \mathrm{MHz}$ for ${ }^{1} \mathrm{H}$ analysis and $75.6 \mathrm{MHz}$ for ${ }^{13} \mathrm{C}$ analysis. Mass spectra were obtained on an Agilent Technology (HP) spectrometer operating at an ionization potential of $70 \mathrm{eV}$. All chemicals were obtained from Merck (Darmastadt, Germany) and Fluka (Buchs, Switzerland) and used without further purification.

\section{General procedure for synthesis of 2-amino-3-cyanopyridine derivatives under solvent free condition}

A mixture of aldehydes (1.0 mmol), malononitrile (1.0 mmol), substituted acetophenone $(1.0 \mathrm{mmol})$, ammonium acetate (1.5 mmol) and cobalt (II) nitrate hexahydrate $(20 \mathrm{~mol} \%)$ as a catalyst was stirred under solvent free condition at $100{ }^{\circ} \mathrm{C}$ for appropriate times. The progress of the reaction was monitored by TLC. After completion of the reaction, the mixture cooled down to room temperature and washed with $\mathrm{EtOH}$ for filtered off the catalyst. The residue product was recrystallized from hot ethanol to afford the pure products $\mathbf{5 a - n}$.

\section{General procedure for four-component synthesis of dihydropyrano[2,3-c]pyrazole derivatives}

A mixture of hydrazine hydrate $(1.0 \mathrm{mmol})$ and ethyl acetoacetate $(1.0 \mathrm{mmol})$ was stirred at room temperature until 3-methyl-2-pyrazoline-5-one was precipitated as white solid and its formation was complete. Then aromatic aldehyde (1.0 mmol), malononitrile (1.0 mmol), cobalt (II) nitrate hexahydrate $(20 \mathrm{~mol} \%)$ and water/ethanol (2:1) was added to the reaction mixture at $70{ }^{\circ} \mathrm{C}$ and stirred for the completion of the reaction. After the completion of the reaction which was monitored by TLC, the reaction mixture was cooled down to room temperature and the product was collected by filtration. The crude product was recrystallized from hot ethanol to afford the pure pyranopyrazole derivatives $\mathbf{8 a - j}$.

\section{General procedure for three-component synthesis of dihydropyrano[2,3-c]pyrazole derivatives}

A mixture of benzaldehyde $(1.0 \mathrm{mmol})$, malononitrile (1.0 mmol), 5-methyl-2-phenyl-2,4-dihydro-3H-pyrazol-3-one $(1.0 \mathrm{mmol})$ and Cobalt (II) nitrate hexahydrate $(20 \mathrm{~mol} \%)$ was added in water/ethanol $(2: 1)$ and then the reaction was stirred at $80{ }^{\circ} \mathrm{C}$. Progress of the reaction was monitored by TLC. After the completion of the reaction, the mixture was cooled to room temperature. Then, the product collected by filtration. The crude principate was recrystallized from hot ethanol to afford the pure pyranopyrazole derivatives 10a-j.

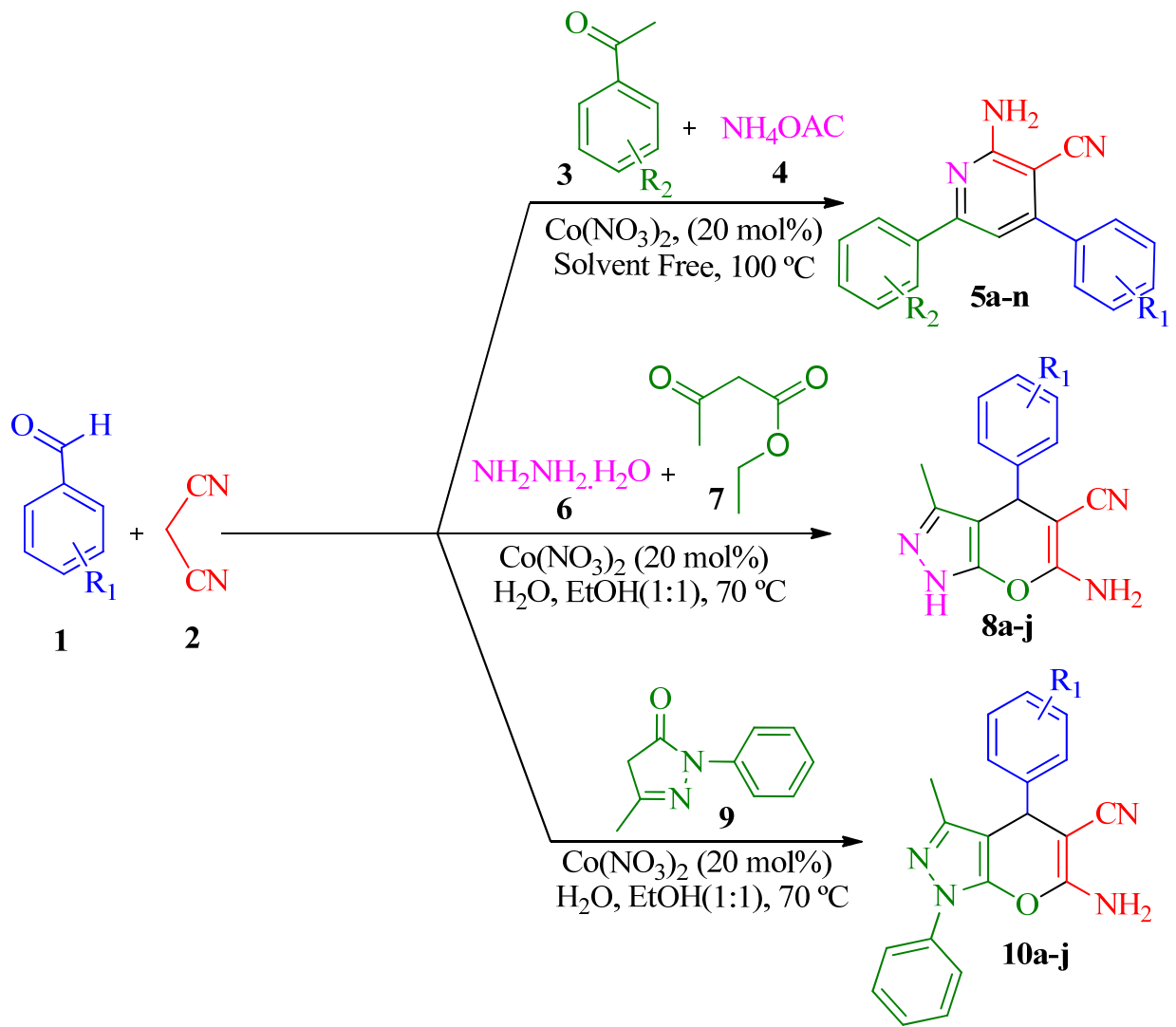

Scheme 1 - Cobalt (II) nitrate hexahydrate catalyzed one-pot synthesis of 4,6-disubstituted 2-aminopyridine-3-carbonitriles and pyranopyrazole derivatives. 
Characterization Data of Selected Compounds

\section{2-Amino-6-(4-chlorophenyl)-4-(3,4-}

\section{dimethoxyphenyl)nicotinonitrile $(5 \mathrm{~m})$ :}

Cream solid, IR (KBr): $v$ 3496, 3369, 2996, 2205, 1628, 1554, 1516, 1258, 808; ${ }^{1} \mathrm{H}$ NMR (300 MHz , DMSO-d6): $\delta$ (ppm) $3.86\left(\mathrm{~s}, 3 \mathrm{H}, \mathrm{OCH}_{3}\right), 3.87\left(\mathrm{~s}, 3 \mathrm{H}, \mathrm{OCH}_{3}\right), 7.02(\mathrm{~s}, 2 \mathrm{H}$, $\left.\mathrm{NH}_{2}\right), 7.13-7.34(\mathrm{~m}, 4 \mathrm{H}, \mathrm{Ar}-\mathrm{H}), 7.58(\mathrm{~d}, J=8.4,2 \mathrm{H}, \mathrm{Ar}-\mathrm{H})$, 8.19 (d, $J=8.1,2 \mathrm{H}, \mathrm{Ar}-\mathrm{H}) ;{ }^{13} \mathrm{C}$ NMR $(75.6 \mathrm{MHz}, \mathrm{DMSO}-\mathrm{d} 6):$ $\delta(\mathrm{ppm})$ 56.1, 87.2, 109.5, 112.1, 112.5, 117.7, 121.6, 129.1, $129.5,135.3,136.9,149.0,150.5,155.3,157.5,161.3 . \mathrm{MS}$ $\mathrm{m} / \mathrm{z}(\%): 182.7(10), 322.2(23), 365.3(\mathrm{M}+, 100)$.

\section{2-Amino-6-(4-chlorophenyl)-4-(3-} nitrophenyl)nicotinonitrile (5n):

White solid, IR (KBr): $v$ 3498, 3398, 2204, 1621, 1579, 1553, 1533, 1349, 826; ${ }^{1} \mathrm{H}$ NMR (300 MHz, DMSO-d6): $\delta(\mathrm{ppm})$ $7.22\left(\mathrm{~s}, 2 \mathrm{H}, \mathrm{NH}_{2}\right), 7.47$ (s, $\left.1 \mathrm{H}, \mathrm{Ar}-\mathrm{H}\right), 7.59$ (d, $J=7.5,2 \mathrm{H}, \mathrm{Ar}-$ $\mathrm{H}), 7.88(\mathrm{t}, J=7.2,1 \mathrm{H}, \mathrm{Ar}-\mathrm{H}), 8.20(\mathrm{~m}, 3 \mathrm{H}, \mathrm{Ar}-\mathrm{H}), 8.41(\mathrm{~d}, J$ $=7.2,1 \mathrm{H}, \mathrm{Ar}-\mathrm{H}), 8.54(\mathrm{~s}, 1 \mathrm{H}, \mathrm{Ar}-\mathrm{H}) ;{ }^{13} \mathrm{C} \mathrm{NMR}(75.6 \mathrm{MHz}$, DMSO-d6): $\delta 87.3,109.7,117.0,123.7,124.8,129.1,129.5$, $130.8,135.6,136.6,138.7,148.3,153.1,158.0,161.2$. MS m/z (\%): 268.7 (10), $304.2(23), 350.1(\mathrm{M}+, 100)$.

\section{6-Amino-1,4-dihydro-3-methyl-4-(4-}

bromophenyl)pyrano[2,3-c]pyrazole-5-carbonitrile (8f):

White powder; M.p.: $236-239{ }^{\circ} \mathrm{C}$; ${ }^{1} \mathrm{H}$ NMR $(300 \mathrm{MHz}$, DMSO-d $\left.d_{6}\right): 1.80\left(\mathrm{~s}, 3 \mathrm{H}, \mathrm{CH}_{3}\right), 4.63(\mathrm{~s}, 1 \mathrm{H}, \mathrm{CH}), 6.96(\mathrm{br}, 2 \mathrm{H}$, $\mathrm{NH}_{2}$ ), 7.14 (d, $\left.J=8 \mathrm{~Hz}, 2 \mathrm{H}, \mathrm{ArH}\right), 7.52$ (d, $\left.J=8 \mathrm{~Hz}, 2 \mathrm{H}, \mathrm{Ar}\right)$, $12.16(\mathrm{~s}, 1 \mathrm{H}, \mathrm{NH})$.

\section{6-Amino-3-methyl-1,4-diphenyl-1,4-} dihydropyrano[2,3,c]pyrazole-5-carbonitrile (10a): White solid; IR (KBr): v 3471, 3324, 2198, 1659 1596, 1516, 753; ${ }^{1} \mathrm{H}$ NMR (300 MHz, DMSO-d6): $\delta$ (ppm) 1.80 (s, 3H,
$\left.\mathrm{CH}_{3}\right), 4.70(\mathrm{~s}, 1 \mathrm{H}, \mathrm{CH}), 7.23\left(\mathrm{~s}, 2 \mathrm{H}, \mathrm{NH}_{2}\right), 7.26-7.40(\mathrm{~m}, 6 \mathrm{H}$, Ar-H), 7.51 (t, 2H, Ar-H), 7.81 (d, 2H, Ar-H).

\author{
6-Amino-1,4-dihydro-4-(4-methoxyphenyl)-3-methyl-1- \\ phenylpyrano[2,3-clpyrazole-5-carbonitrile (10c): \\ White powder; M.p.: $174-177{ }^{\circ} \mathrm{C}$; ${ }^{1} \mathrm{H}$ NMR $(300 \mathrm{MHz}$, \\ DMSO-d6): $1.80\left(\mathrm{~s}, 3 \mathrm{H}, \mathrm{CH}_{3}\right), 3.64\left(\mathrm{~s}, 3 \mathrm{H}, \mathrm{OCH}_{3}\right), 4.64(\mathrm{~s}$, \\ $1 \mathrm{H}, \mathrm{CH}), 6.92(\mathrm{~d}, J=8 \mathrm{~Hz}, 2 \mathrm{H}, \mathrm{Ar}), 7.19\left(\mathrm{~s}, 2 \mathrm{H}, \mathrm{NH}_{2}\right), 7.20$ \\ $(\mathrm{d}, J=8 \mathrm{~Hz}, 2 \mathrm{H}, \mathrm{ArH}), 7.33(\mathrm{t}, J=8 \mathrm{~Hz}, 1 \mathrm{H}), 7.50(\mathrm{t}, J=8$ \\ $\mathrm{Hz}, 2 \mathrm{H}, \mathrm{ArH}), 7.80$ (d, $J=8 \mathrm{~Hz}, 2 \mathrm{H})$.
}

\section{RESULTS AND DISCUSSION}

As a trial model, the reaction between acetophenone, benzaldehyde, malononitrile and ammonium acetate in equimolar amounts was selected for the synthesis of 4,6-disubstituted 2-aminopyridine3-carbonitriles. At first, the effects of solvents were evaluated and it was found that the performance of reaction under solvent-free conditions have higher priority (Table 1 , entry 1 ). Then the effects of different proportion of catalyst at different temperatures were evaluated. As shown in (Table 2, entry 7), the best result obtained with $20 \mathrm{~mol} \%$ of cobalt (II) nitrate hexahydrate at $100^{\circ} \mathrm{C}$.

Using these optimized experimental conditions, a number of aromatic aldehydes and various acetophenone were reacted with malononitrile and ammonium acetate to led product 5a-n (Table 3).

Table 1

Optimization of solvent for the synthesis of 2-aminopyridine-3-carbonitriles

\begin{tabular}{ccccc}
\hline Entry & Solvent & Temperature $\left({ }^{\circ} \mathrm{C}\right)$ & Time $(\mathrm{min})$ & Yield $(\%)$ \\
\hline $\mathbf{1}$ & Solvent Free & $\mathbf{1 0 0}$ & $\mathbf{2 0}$ & $\mathbf{9 0}$ \\
2 & $\mathrm{H}_{2} \mathrm{O}:$ EtOH $(1: 1)$ & 80 & 60 & 49 \\
3 & $\mathrm{EtOH}$ & 70 & 60 & 43 \\
4 & $\mathrm{H}_{2} \mathrm{O}$ & 100 & 80 & 38 \\
\hline
\end{tabular}

Table 2

Effect of the amount of catalyst and temperature on the model reaction

\begin{tabular}{|c|c|c|c|c|}
\hline & & & $\mathrm{NH}_{2}$ & \\
\hline Entry & Catalyst $(\mathrm{mol} \mathrm{\%})$ & Temperature $\left({ }^{\circ} \mathrm{C}\right)$ & Time (min) & Yield (\%) \\
\hline 1 & 20 & 30 & 150 & 60 \\
\hline 2 & 20 & 60 & 65 & 77 \\
\hline 3 & 20 & 70 & 60 & 77 \\
\hline 4 & 20 & 80 & 50 & 80 \\
\hline 5 & 20 & 90 & 40 & 88 \\
\hline 7 & 20 & 100 & 20 & 90 \\
\hline 8 & 20 & 110 & 30 & 82 \\
\hline 9 & 5 & 100 & 40 & 69 \\
\hline 10 & 10 & 100 & 35 & 74 \\
\hline 11 & 15 & 100 & 30 & 81 \\
\hline 12 & 25 & 100 & 25 & 83 \\
\hline
\end{tabular}


Table 3

The synthesis of 2-amino-3-cyanopyridine derivatives under solvent-free condition

\begin{tabular}{|c|c|c|c|c|c|c|c|}
\hline Entry & $\mathbf{R}_{1}$ & $\mathbf{R}_{\mathbf{2}}$ & Product & $\begin{array}{l}\text { Time } \\
\text { (min) }\end{array}$ & Yield (\%) & M.p $\left({ }^{\circ} \mathrm{C}\right)$ & $\begin{array}{l}\text { M.p }\left({ }^{\circ} \mathrm{C}\right) \\
\text { [Lit.]Ref. }\end{array}$ \\
\hline 1 & $\mathrm{H}$ & $\mathrm{H}$ & $5 a$ & 20 & 91 & $183-185$ & $186-188^{24}$ \\
\hline 2 & $4-\mathrm{F}$ & $\mathrm{H}$ & $5 b$ & 20 & 89 & $149-148$ & $148-150^{24}$ \\
\hline 3 & $4-\mathrm{Cl}$ & $\mathrm{H}$ & $5 c$ & 15 & 92 & $186-188$ & $188-190^{25}$ \\
\hline 4 & $4-\mathrm{Br}$ & $\mathrm{H}$ & $5 d$ & 25 & 93 & $226-228$ & $225-228^{26}$ \\
\hline 5 & $3-\mathrm{NO}_{2}$ & $\mathrm{H}$ & $5 e$ & 30 & 91 & $198-200$ & $200-202^{24}$ \\
\hline 6 & $4-\mathrm{Me}$ & $\mathrm{H}$ & $5 \mathrm{~g}$ & 25 & 83 & $228-230$ & $231-233^{24}$ \\
\hline 7 & 4-OMe & $\mathrm{H}$ & $5 \mathrm{~h}$ & 35 & 83 & $180-181$ & $181-182^{25}$ \\
\hline 8 & $2-\mathrm{Cl}$ & $\mathrm{H}$ & $5 i$ & 45 & 85 & 190-192 & $193-196^{24}$ \\
\hline 9 & $\mathrm{H}$ & $4-\mathrm{Cl}$ & $\mathbf{5 j}$ & 15 & 92 & $237-240$ & $238-240^{24}$ \\
\hline 10 & 4-OMe & $4-\mathrm{Cl}$ & $5 \mathbf{k}$ & 30 & 87 & $205-206$ & $204-206^{26}$ \\
\hline 11 & $4-\mathrm{OMe}$ & $4-\mathrm{Br}$ & 51 & 35 & 89 & $178-180$ & $180-182^{26}$ \\
\hline 12 & $3,4-\mathrm{OMe}$ & $4-\mathrm{Cl}$ & $5 \mathrm{~m}$ & 40 & 82 & $207-208$ & This work \\
\hline 13 & $3-\mathrm{NO}_{2}$ & 4-Cl & $5 n$ & 20 & 90 & $277-279$ & This work \\
\hline
\end{tabular}

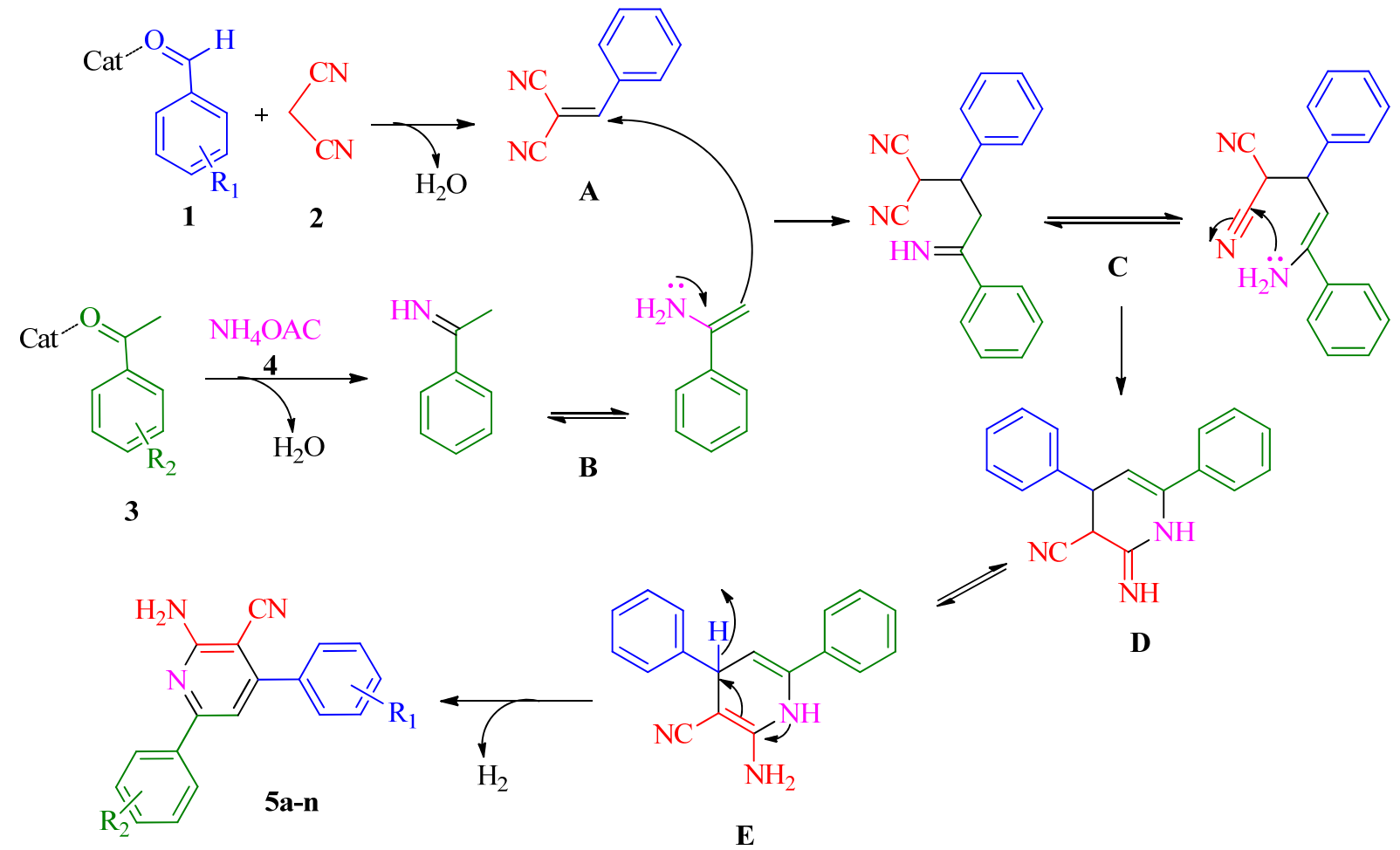

Scheme 2 - The proposed mechanism for the synthesis of 2-amino-3-cyanopyridines.

A mechanistic explanation for the formation of 2-amino-3-cyanopyridines is illustrated in scheme 2. First, the Knoevenagel condensation between active aldehyde $\mathbf{1}$ and malononitrile $\mathbf{2}$ generate the arylidene malononitrile $\mathbf{A}$ that reacts via Michael addition with enamine $\mathbf{B}$ from the coupling of acetophenone $\mathbf{3}$ and ammonium acetate $\mathbf{4}$ to give intermediate $\mathbf{C}$. Finally, intermediate $\mathbf{C}$ undergoes subsequent stages intramolecular cyclization/isomerization/aromatization to afford the desired product (5a-n).

In another study for the synthesis of dihydropyrano[2,3-c]pyrazoles, we selected the reaction of ethyl acetoacetate $(1.0 \mathrm{mmol})$, hydrazine hydrate $(1.0 \mathrm{mmol})$, malononitrile $(1.0 \mathrm{mmol})$, and benzaldehyde $(1.0 \mathrm{mmol})$ as a model system and the effect of amount of solvent, catalyst and temperature was envisaged. First, the effects of solvents (ethanol and aqueous ethanol) were evaluated and it was found that the rate 2:1 $\mathrm{H}_{2} \mathrm{O} / \mathrm{EtOH}$ is better than other rates (Table 4, entry 3 ). Optimization of the other reaction conditions demonstrated that the best results were gained when the reaction was performed at $70{ }^{\circ} \mathrm{C}$ in the presence of cobalt (II) nitrate hexahydrate (20 mol\%) (Table 5, entry 4). 
Table 4

Optimization of solvent for the synthesis of dihydropyrano[2,3-c]pyrazoles at $70{ }^{\circ} \mathrm{C}$

\begin{tabular}{cccc}
\hline Entry & Solvent & Time (min) & Yield (\%) \\
\hline 1 & $\mathrm{H}_{2} \mathrm{O}:$ EtOH $(1: 2)$ & 20 & 81 \\
2 & $\mathrm{H}_{2} \mathrm{O}:$ EtOH $(1: 1)$ & 15 & 70 \\
3 & $\mathbf{H}_{2} \mathbf{O}:$ EtOH $(2: 1)$ & $\mathbf{8}$ & $\mathbf{9 1}$ \\
4 & $\mathrm{EtOH}$ & 25 & 47 \\
5 & $\mathrm{H}_{2} \mathrm{O}$ & 17 & 53 \\
\hline
\end{tabular}

Table 5

Effect of the amount of catalyst and temperature on the model reaction

\begin{tabular}{ccccc}
\hline Entry & Catalyst (mol \%) & Time (min) & Temperature $\left({ }^{\circ} \mathbf{C}\right)$ & Yield (\%) \\
\hline 1 & 20 & 45 & 30 & 61 \\
2 & 20 & 35 & 50 & 66 \\
3 & 20 & 25 & 60 & 70 \\
$\mathbf{4}$ & $\mathbf{2 0}$ & $\mathbf{8}$ & $\mathbf{7 0}$ & $\mathbf{9 1}$ \\
5 & 20 & 50 & 80 & 78 \\
6 & 5 & 27 & 70 & 76 \\
7 & 10 & 18 & 70 & 81 \\
8 & 15 & 14 & 70 & 91 \\
9 & 20 & 8 & 70 & 86 \\
10 & 25 & 25 & 70 & \\
\hline
\end{tabular}

Table 6

Synthesis of dihydropyrano[2,3-c]pyrazole by reaction of hydrazine hydrate, ethyl acetoacetate, aromatic aldehyde and malononitrile in the presence of cobalt (II) nitrate hexahydrate $(20 \mathrm{~mol} \%)$ in water/ethanol $(2: 1)$ at $70{ }^{\circ} \mathrm{C}$

\begin{tabular}{ccccccc}
\hline Entry & $\mathbf{R}_{\mathbf{1}}$ & Product & Time (min) & Yield (\%) & M.p $\left(^{\circ} \mathbf{C}\right)$ & M.p $\left({ }^{\circ} \mathbf{C}\right)[$ Lit. $]$ \\
\hline 1 & & & & & & \\
2 & $\mathrm{H}$ & $\mathbf{8 a}$ & 8 & 91 & $243-245$ & $247-248^{27}$ \\
3 & $4-\mathrm{Cl}$ & $\mathbf{8 b}$ & 6 & 89 & $233-235$ & $234-236^{28}$ \\
4 & $4-\mathrm{OMe}$ & $\mathbf{8 c}$ & 17 & 91 & $210-211$ & $210-212^{29}$ \\
5 & $3-\mathrm{NO}_{2}$ & $\mathbf{8 d}$ & 12 & 90 & $191-193$ & $193-195^{29}$ \\
6 & $2-\mathrm{Cl}$ & $\mathbf{8 e}$ & 15 & 89 & $141-144$ & $145-147^{28}$ \\
7 & $4-\mathrm{Br}$ & $\mathbf{8 f}$ & 6 & 93 & $176-179$ & $178-180^{29}$ \\
8 & $4-\mathrm{NO}_{2}$ & $\mathbf{8 g}$ & 10 & 90 & $250-252$ & $250-252^{27}$ \\
9 & $4-\mathrm{Me}^{27}$ & $\mathbf{8 h}$ & 12 & 91 & $203-206$ & $206-208^{28}$ \\
10 & $4-\mathrm{OH}^{2}-\mathrm{OCH}_{3}$ & $\mathbf{8 i}$ & 17 & 88 & $230-233$ & $235-237^{30}$ \\
\hline
\end{tabular}

\section{Table 7}

Synthesis of dihydropyrano[2,3-c]pyrazoles by reaction of 5-methyl-2-phenyl-2,4-dihydro-3H-pyrazol-3-one, aromatic aldehyde and malononitrile in the presence of cobalt (II) nitrate hexahydrate $(20 \mathrm{~mol} \%)$ in water/ethanol $(2: 1)$ at $70{ }^{\circ} \mathrm{C}$

\begin{tabular}{ccccccc}
\hline Entry & $\mathbf{R}_{\mathbf{1}}$ & Product & Time (min) & Yield (\%) & M.p $\left({ }^{\circ} \mathbf{C}\right)$ & M.p $\left({ }^{\circ} \mathbf{C}\right)[\mathbf{L i t}]$. \\
\hline 1 & $\mathrm{H}$ & $\mathbf{1 0 a}$ & 10 & 93 & $167-169$ & $169-171^{31}$ \\
2 & $4-\mathrm{Cl}$ & $\mathbf{1 0 b}$ & 6 & 91 & $170-172$ & $173-175^{32}$ \\
3 & $4-\mathrm{OMe}$ & $\mathbf{1 0 c}$ & 17 & 89 & $169-172$ & $174-176^{32}$ \\
4 & $3-\mathrm{NO}_{2}$ & $\mathbf{1 0 d}$ & 12 & 90 & $189-190$ & $190-192^{33}$ \\
5 & $2-\mathrm{Cl}$ & $\mathbf{1 0 e}$ & 10 & 89 & $139-142$ & $144-146^{32}$ \\
6 & $4-\mathrm{Br}$ & $\mathbf{1 0 f}$ & 8 & 93 & $176-177$ & $177-179^{32}$ \\
7 & $4-\mathrm{NO}_{2}$ & $\mathbf{1 0 g}$ & 10 & 90 & $197-199$ & $197-199^{32}$ \\
8 & $4-\mathrm{Me}$ & $\mathbf{1 0 h}$ & 12 & 91 & $172-175$ & $174-177^{34}$ \\
9 & $4-\mathrm{OH}$ & $\mathbf{1 0 i}$ & 35 & 86 & $208-211$ & $208-210^{33}$ \\
10 & $2,4-\mathrm{diCl}$ & $\mathbf{1 0 j}$ & 15 & 83 & $177-180$ & $181-183^{32}$ \\
\hline
\end{tabular}




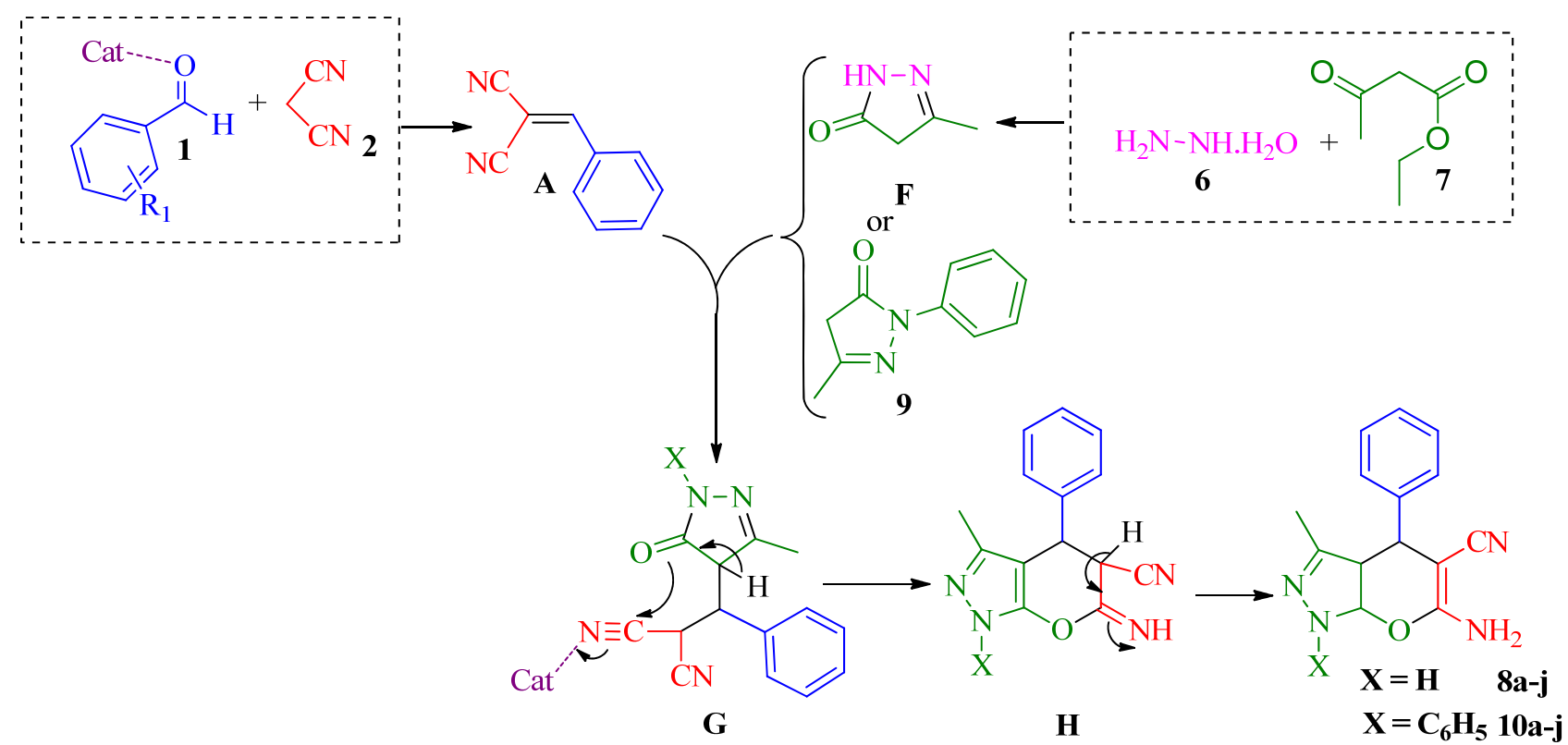

Scheme 3 - The proposed mechanism for the synthesis of dihydropyrano[2,3-c]pyrazoles.

After optimizing the reaction conditions, we evaluated the range and feasibility of reactions using a various aryl aldehydes. Forasmuch as, the synthesis of other pyranopyrazole derivatives of three-component reaction as same conditions has resulted in good yields, so we have accomplished two reactions at the similar conditions. As shown in Table 6 and 7, two reactions tolerated both electron-withdrawing and electron-donating groups on the aldehyde aromatic rings.

For the synthesis of dihydropyrano[2,3-c]pyrazoles is proposed that the arylidene malononitrile $\mathbf{A}$ to generate in situ via Knoevenagel condensation active aldehyes $\mathbf{3}$ and malononitrile 2. Michael addition of arylidene malononitrile $\mathbf{A}$ and 5-methyl2-phenyl-2,4-dihydro-3 $H$-pyrazol-3-one $\mathbf{9}$ or pyrazolone $\mathbf{F}$ (from the reaction between hydrazine hydrate $\mathbf{6}$ and ethyl acetoacetate 7) gives the acyclic adduct products $\mathbf{G}$, which undergoes intramolecular cyclization and tautomerization to afford the corresponding products (Scheme 3).

\section{CONCLUSIONS}

In compendium, we expanded three simple and efficient protocol for one-pot synthesis of 2-amino3 -cyanopyridine and dihydropyrano[2,3-c]pyrazole derivatives in the presence of cobalt (II) nitrate hexahydrate. Good to excellent yields, use of commercially available low-cost starting materials, simplicity of operation, cleaner reaction profile are the key advantages of the present methodologies.
Acknowledgment: We gratefully appreciate financial support from the Research Council of University of Sistan and Baluchestan.

\section{REFERENCES}

1. S.S. Murphre, "Heterocyclic dyes: Preparation, properties, and applications", in "Progress in Heterocyclic Chemistry", Elsevier: Amsterdam, The Netherlands, 2011, 22, p. 21-58.

2. C. Lamberth and J. Dinges, "The significance of heterocycles for pharmaceuticals and agrochemicals", in "Bioactive Heterocyclic Compound Classes: Agrochemicals", Wiley-VCH Verlag GmbH \& Co. KGaA: Weinheim, Germany, 2012.

3. S. Maddila, R. Pagadala and S.B. Jonnalagadda, Lett. Org. Chem., 2013, 10, 693-714.

4. M. Mantri, O. De Graaf, J. Van Veldhoven, A. Goblyos, J.K. Von Frijtag Drabbe Kunzel, T. Mulder-Krieger, R. Link, H. De Vries, M.W. Beukers, J. Brussee and A.P. Ijzerman, J. Med. Chem., 2008, 51, 4449-4455.

5. T. Murata, M. Shimada, H, Kadono, S. Sakakibara, T. Yoshino, T. Masuda, M. Shimazaki, T. Shintani, K. Fuchikami, K.B. Bacon and T.B.Z. Lowinger. Bioorg. Med. Chem. Lett., 2004, 14, 4013-4017.

6. J. Deng, T. Sanchez, L.Q. Al-Mawsawi, R. Dayam, R.A. Yunes, A. Garofalo, M.B. Bolger and N. Neamati, Bioorg. Med. Chem., 2007, 15, 4985-5002.

7. K. Gobis, H. Foks, A. Kędzia, M. Wierzbowska and Z. Zwolska, J. Heterocycl. Chem., 2009, 46, 1271-1279.

8. F. Zhang, Y. Zhao, L. Sun, L. Ding, Y. Gu and P. Gong, Eur. J. Med. Chem., 2011, 46, 3149-3157.

9. A.A. Bekhit and A.M. Baraka, Eur. J. Med. Chem., 2005, 40, 1405-1413.

10. T. Murata, M. Shimada, S. Sakakibara, T. Yoshino, H. Kadono, T. Masuda, M. Shimazaki, T. Shintani, K. Fuchikami, K. Sakai, H. Inbe, K. Takeshita, T. Niki, M. Umeda, K.B. Bacon, K.B. Ziegelbauer and T.B. Lowinger, Bioorg. Med. Chem. Lett., 2003, 13, 913-918. 
11. H. Kiyani, F. Albooyeh and S. Fallahnezhad, J. Mol. Struct., 2015, 1091, 163-169.

12. A. Ansari, A. Ali and M. Asif, New J. Chem., 2017, 41, $16-41$.

13. B.P. Bandgar, H.V. Chavan, L.K. Adsul, V.N. Thakare, S.N. Shringare, R. Shaikh and R.N. Gacche, Bioorg. Med. Chem. Lett., 2013, 23, 912-919.

14. K.M. Kasiotis, E.N. Tzanetou and S.A. Haroutounian, Front. Chem., 2014, 2, 78.

15. M.J. Genin, C. Biles, B.J. Keiser, S.M. Poppe, S.M. Swaney, W.G. Tarpley, Y. Yagi and D.L. Romero, $J$. Med. Chem., 2000, 43, 1034-1040.

16. F. Abrigach and R. Touzani, Med. Chem (Los Angeles)., 2016, 6, 292-298.

17. A.V. Stachulski, N.G. Berry, A.C.L. Low, S.L. Moores, E. Row, D.C. Warhurst, I.S. Adagu and J.F. Rossignol, $J$. Med. Chem., 2006, 49, 1450-1454.

18. C.J. Shishoo, M.B. Devani, V.S. Bhadti, S. Ananthan and G.V. Ullas, Tetrahedron Lett., 1983, 24, 4611-4612.

19. M. Fatahpour, F. Noori Sadeh, N. Hazeri, M.T. Maghsoodlou and M. Lashkari, J. Iran. Chem. Soc., 2017, 14, 1945-1956.

20. M. Fatahpour, F. Noori Sadeh, N. Hazeri, M.T. Maghsoodlou, M.S. Hadavi and S. Mahnaei, J. Saudi Chem. Soc., 2017, 21, 998-1006.

21. M. Fatahpour, N. Hazeri, M.T. Maghsoodlou and M. Lashkari, Polycycl. Aromat. Comp., 2017, DOI: $10.1080 / 10406638.2017 .1326948$.

22. P. Dastoorani, M. T. Maghsoodlou, M. A. Khalilzadeh, and E. Sarina, Tetrahedron Lett., 2016, 57, 314-316.
23. S.S. Sajadikhah, M.T. Maghsoodlou and N. Hazeri, Chin. Chem. Lett., 2014, 25, 58-60.

24. M.A. Zolfigol, M. Kiafar, M. Yarie, A.A. Taherpour and M. Saeidi-Rad, $R S C A d v$., 2016, 6, 50100-50111.

25. M. Abdollahi-Alibeik, N. Sadeghi-Vasafi, A. Moaddeli and A. Rezaeipoor-Anari, Res. Chem. Intermed., 2016, 42, 2867-2881.

26. S.S. Mansoor, K. Aswin, K. Logaiya, P.N. Sudhan and S. Malik, Res. Chem. Intermed., 2014, 40, 871-885.

27. C.F. Zhou, J.J. Li, and W.k. Su, Chin. Chem. Lett., 2016, 27, 1686-1690.

28. H. Mecadon, M. R. Rohman, M. Rajbangshi and B. Myrboh, Tetrahedron Lett., 2011, 52, 2523-2525.

29. M. Zakeri, M. M. Nasef, T. Kargaran, A. Ahmad, E. Abouzari-Lotf and J. Asadi, Res. Chem. Intermed,. 2017, 43, 717-728.

30. M. A. Zolfigol, M. Tavasoli, A.R. Moosavi-Zare, P. Moosavi, H.G. Kruger, M. Shiri and V. Khakyzadeh, RSC Adv., 2013, 3, 25681-25685.

31. S. R. Mandha, S. Siliveri, M. Alla, V. R. Bommena, M. R. Bommineni and S. Balasubramanian, Bioorg. Med. Chem. Lett., 2012, 22, 5272-5278.

32. J. Albadi, A. Mansournezhad and Z. Derakhshandeh, Chin. Chem. Lett., 2013, 24, 821-824.

33. J. M. Khurana, B. Nand, and S. Kumar, Synth. Commun., 2011, 41, 405-410.

34. B. Maleki, N. Nasiri, R. Tayebee, A. Khojastehnezhad and H. A. Akhlaghi, RSC Adv., 2016, 6, 79128-79134. 
